\title{
Evaluando características del agente software
}

\author{
Evaluation of the characteristics of software agent \\ Héctor Soza Pollman ${ }^{1}$ \\ Recibido 25 de febrero de 2014, aceptado 30 de abril de 2014 \\ Received: February 25, 2014 Accepted: April 30, 2014
}

\begin{abstract}
RESUMEN
Este trabajo es el resultado de una investigación para evaluar características de un agente software. Para ello se consideran las tres características más relevantes de un agente software: su habilidad social, autonomía y proactividad. Se definen atributos medibles por cada característica y se proponen medidas que permitan evaluar cada atributo y, a partir de estos valores, obtener el valor de las características consideradas. Para validarlas se aplican estas medidas a un caso de estudio.
\end{abstract}

Palabras clave: Agente software, características del agente, habilidad social, autonomía, proactividad.

\begin{abstract}
This work is the result of an investigation in order to evaluate characteristics of a software agent. For this purpose, the three most important characteristics of a software agent were considered: its social ability, autonomy and pro-activity. Measurable attributes are defined for each feature and measures to evaluate each attribute, and from these values, obtain the value of the characteristics considered are proposed. These measures were applied to a case study for evaluation purpose.
\end{abstract}

Keywords: Software agent, software characteristics, social hability, autonomy, pro-activity.

\section{INTRODUCCIÓN}

La calidad de un producto software se refiere a los factores que contribuyen a la satisfacción completa de las necesidades de un usuario u organización relacionadas con ese producto [1]. En el desarrollo de la Ingeniería Informática se han presentado varios modelos de calidad del software, como el modelo procedural [2], el orientado a objetos [3] y el estándar de calidad internacional establecido por ISO/IEC [4] entre otros, pero no un modelo que mida la calidad de un agente software.

Para evaluar el agente software se han investigado trabajos publicados en relación con medidas para evaluarlo, los que consideran principalmente la adopción de medidas de otros paradigmas, como el procedural y orientado a objetos, ya que hay características de programación en común con el software orientado a agentes, como son la programación modular y la encapsulación [5-6]. Otros trabajos han propuesto algunas medidas nuevas pero no relacionadas con atributos específicos del agente software [7] y han desarrollado test orientados a evaluar metodologías orientadas a agentes [8].

Es manifiesto entonces que el aporte de este trabajo está en la necesidad de evaluar el agente software, aportando medidas adecuadas que consideren las características relevantes de estos agentes.

Para evaluar la calidad de un software, ISO/IEC propuso en 2001 descomponer esta calidad en tres niveles jerárquicos: características (se corresponden con propiedades que debe verificar el software), subcaracterísticas o atributos (son cualidades medibles

1 Escuela de Ingeniería. Universidad Católica del Norte. Larrondo 1281. Coquimbo, Chile. E-mail: hsoza@ucn.cl 
que influyen en cada característica) y medidas (son las métricas que permiten evaluar los atributos) [4]. Basados en esta descomposición se ha planteado este trabajo de investigación para evaluar características de un agente software.

En relación con las características de un agente software, se ha realizado el estudio de las características de habilidad social, autonomía y proactividad, con sus atributos y medidas asociadas, por considerar que son las más representativas del agente software [9-12]. El objetivo de este trabajo de investigación es proponer atributos asociados a las características mencionadas del agente software y un conjunto de medidas que permitan evaluar dichos atributos [13].

El resto del artículo está estructurado como sigue. Se definen las características de un agente y se presentan los atributos relacionados con la habilidad social, autonomía y la proactividad. Luego se presentan trabajos relacionados con el desarrollo de medidas para evaluar características del agente software. A continuación se describen medidas para evaluar estas características y se presenta su aplicación a un caso de estudio. Se finaliza el artículo con una serie de conclusiones y líneas de investigación futura.

\section{CARACTERÍSTICAS DEL AGENTE SOFTWARE}

De acuerdo con McCall [2] e ISO/IEC [4], para medir la calidad de un producto software se deben definir tres aspectos del producto: sus características, sus subcaracterísticas (llamados atributos) y las medidas de estos atributos.

Las características consideradas en este trabajo como más representativas de un agente software son su habilidad social, autonomía y proactividad [13].

La habilidad social es la capacidad del agente de interactuar con otros agentes para alcanzar sus objetivos [16-17]. La autonomía es la capacidad del agente de operar por sí mismo sin la necesidad de guía humana o la intervención de elementos externos controlando su estado interno y acciones $[5,18]$. La proactividad es la capacidad del agente de tener una conducta dirigida por objetivos tomando la iniciativa para alcanzar sus metas $[16,7,12]$.

La importancia de la habilidad social está en que para alcanzar sus objetivos el agente debe ser capaz de comunicarse, negociar y cooperar con los demás agentes del sistema [16].

La importancia de la autonomía radica en que para lograr un objetivo el agente debe tener la posibilidad de decidir, no solo cómo alcanzar un objetivo, sino también que los objetivos deben ser buscados sobre la base de un interés generado endógenamente [19], para lo que debe controlar su estado interno, tener independencia y la capacidad de adaptarse a las necesidades de su entorno [18].

Finalmente, la importancia de la proactividad está en que implica que el agente ejecute acciones por iniciativa propia para alcanzar sus objetivos, interactuando con los agentes en el entorno y reaccionando a los estímulos que aparecen en este entorno, por lo que debe tener la capacidad de evaluar su entorno y decidir qué acción tomar [20].

\section{TRABAJO RELACIONADO}

Considerando la investigación desarrollada sobre las características y medidas de calidad de un agente software, pocos estudios en la literatura se han enfocado en proponer medidas relacionadas con las características de habilidad social, autonomía y proactividad de un agente software y pocos definen un conjunto de medidas específicas para evaluar estas características.

Dumke, Koeppe y Wille, proponen medir la habilidad social del agente usando la complejidad del agente como un modo de conocer el grado de las dimensiones organizacionales del agente (como son las sociales, relacionales, del entorno y personales). No obstante, en sus publicaciones no mencionan cómo medir esta complejidad [5].

Cernuzzi y Rossi propusieron un marco de trabajo para realizar una evaluación del análisis y diseño de métodos de modelamiento orientado a agentes. Ellos evalúan la autonomía de un agente considerando cuándo o no las técnicas de modelamiento chequean si los agentes tienen control sobre su estado interno y su conducta [14].

En 2004, Shin presentó los resultados de adaptar algunas medidas de los paradigmas procedural y orientado a objetos, al software orientado a agentes, así como de agregar medidas específicas para este 
paradigma. Propuso que el acoplamiento entre objetos es una medida para evaluar la habilidad social de un agente, y que la complejidad del agente puede ser considerada como una medida de su autonomía. Propuso además medir la proactividad considerando la frecuencia de descubrimiento de conocimiento basado en la actualización del conocimiento interno del agente, para lo que considera la cantidad de mensajes que el agente usa para descubrir conocimiento de los agentes en su entorno. En su trabajo no define atributos para evaluar estas características, solo define medidas para valorar algunos aspectos de estas características del software del agente [7].

Huber estudió la autonomía de los agentes software. Propone medir la dependencia social para evaluar la autonomía. Para calcular un valor global de la autonomía de un agente software Huber combina el valor de la autonomía de integridad social y el valor de la autonomía de la dependencia [15].

El estado del arte pone de manifiesto la necesidad de proponer medidas que permitan evaluar las características más relevantes del agente software, con medidas adecuadas que consideren los atributos relacionados con estas características.

\section{ATRIBUTOS Y MEDIDAS}

Las medidas que se proponen para cada atributo son resultado de la investigación en agentes software. Algunas fueron seleccionadas a partir de los paradigmas procedural y orientado a objetos, y otras son nuevas medidas especialmente diseñadas para evaluar estos atributos [13].

\section{Atributos y medidas de habilidad social}

La habilidad social en los agentes se entiende como la competencia que tienen en el momento de intercambiar información entre ellos (comunicación), la destreza en la colaboración conjunta (cooperación) y la habilidad para llegar a un acuerdo sobre el camino a seguir para alcanzar sus objetivos (negociación) [17].

El atributo comunicación se identifica con la habilidad de recibir y enviar mensajes por parte del agente para alcanzar sus objetivos. Se ha determinado que una buena comunicación del agente puede ser evaluada considerando la cantidad de mensajes invocado en respuesta a un mensaje recibido por el agente, la cantidad de mensajes entrantes y salientes recibidos y enviados por el agente para mantener un enlace de comunicación significativo o realizar algunos objetivos, y el tamaño de los mensajes enviados por el agente durante la ejecución [7, 17].

El atributo cooperación se identifica con la habilidad del agente para responder a los servicios solicitados por otros agentes y ofrecer servicios a otros agentes. Se ha determinado que una buena cooperación puede ser evaluada considerando la capacidad del agente de aceptar o rechazar los servicios solicitados por otros agentes, y la capacidad del agente para ofrecer servicios [16-17].

El atributo negociación se identifica con la habilidad del agente para establecer compromisos, resolver conflictos y alcanzar acuerdos con otros agentes para asegurar el cumplimiento de sus objetivos. Por lo tanto este atributo puede ser evaluado considerando la cantidad de objetivos logrados, la cantidad de mensajes enviados por el agente cuando otro agente requiere un servicio de él, y la cantidad de mensajes enviados por el agente cuando solicita un servicio a otros agentes [16-17]. La Tabla 1 presenta las nueve medidas propuestas para evaluar habilidad social de un agente software y sus definiciones.

\section{Atributos y medidas de autonomía}

La autonomía en los agentes se entiende como la capacidad del agente para controlar sus propios actos (autocontrol), la habilidad para manejar su comportamiento y otra información relacionada con sus acciones (independencia funcional) y la aptitud a la hora de autoajustarse y adaptarse a nuevos requisitos (capacidad de evolución) [18].

El atributo autocontrol se identifica con el hecho que el agente es capaz de operar por sí mismo, sin la necesidad de apoyo humano o la intervención de elementos externos, para alcanzar sus objetivos [23], y con el nivel de control que el agente tiene sobre su propio estado y comportamiento [5].

El atributo independencia funcional se identifica con las tareas ejecutivas que requieren una acción que el agente ha de realizar en nombre de cualquiera de los usuarios que representa o de otros agentes [16, 7]. Un buen nivel de independencia funcional indica que el agente no tiene que realizar muchas tareas 
Tabla 1. Medidas característica habilidad social.

\begin{tabular}{|c|c|c|}
\hline \multicolumn{3}{|c|}{ Medidas de habilidad social } \\
\hline \multirow{4}{*}{ Comunicación } & $\begin{array}{l}\text { Respuestas por } \\
\text { mensaje }\end{array}$ & $\begin{array}{l}\text { Mide la cantidad } \\
\text { de mensajes que se } \\
\text { envían en respuesta } \\
\text { a un mensaje } \\
\text { recibido por el } \\
\text { agente }\end{array}$ \\
\hline & $\begin{array}{l}\text { Tamaño } \\
\text { promedio del } \\
\text { mensaje }\end{array}$ & $\begin{array}{l}\text { Mide la influencia } \\
\text { del tamaño de datos } \\
\text { de los mensajes } \\
\text { enviados por } \\
\text { el agente en su } \\
\text { comunicación } \\
\end{array}$ \\
\hline & $\begin{array}{l}\text { Cantidad } \\
\text { de mensajes } \\
\text { recibidos }\end{array}$ & $\begin{array}{l}\text { Mide la relación } \\
\text { de los mensajes } \\
\text { recibidos en la } \\
\text { comunicación del } \\
\text { agente durante su } \\
\text { tiempo de vida }\end{array}$ \\
\hline & $\begin{array}{l}\text { Cantidad } \\
\text { de mensajes } \\
\text { enviados }\end{array}$ & $\begin{array}{l}\text { Mide la relación } \\
\text { entre los mensajes } \\
\text { enviados por el } \\
\text { agente con la } \\
\text { comunicación del } \\
\text { agente durante su } \\
\text { tiempo de ejecución }\end{array}$ \\
\hline \multirow[t]{2}{*}{ Cooperación } & $\begin{array}{l}\text { Solicitudes } \\
\text { de servicio } \\
\text { rechazadas } \\
\text { por el agente }\end{array}$ & $\begin{array}{l}\text { Mide la influencia } \\
\text { del porcentaje } \\
\text { de los servicios } \\
\text { rechazados por el } \\
\text { agente cuando otros } \\
\text { agentes requieren } \\
\text { sus servicios } \\
\end{array}$ \\
\hline & $\begin{array}{l}\text { Cantidad } \\
\text { de servicios } \\
\text { ofrecidos por } \\
\text { el agente }\end{array}$ & $\begin{array}{l}\text { Mide la cantidad } \\
\text { de servicios que } \\
\text { el agente publicita } \\
\text { en el directorio de } \\
\text { páginas amarillas de } \\
\text { su entorno }\end{array}$ \\
\hline \multirow{3}{*}{ Negociación } & $\begin{array}{l}\text { Objetivos } \\
\text { alcanzados } \\
\text { por el agente }\end{array}$ & $\begin{array}{l}\text { Mide la eficiencia de } \\
\text { la negociación del } \\
\text { agente para alcanzar } \\
\text { sus objetivos, con el } \\
\text { apoyo de los demás } \\
\text { agentes del entorno }\end{array}$ \\
\hline & $\begin{array}{l}\text { Mensajes por } \\
\text { un servicio } \\
\text { solicitado }\end{array}$ & $\begin{array}{l}\text { Mide la influencia } \\
\text { de la cantidad } \\
\text { de mensajes } \\
\text { intercambiados por } \\
\text { el agente al realizar } \\
\text { una negociación, } \\
\text { cuando otro agente } \\
\text { está solicitando sus } \\
\text { servicios }\end{array}$ \\
\hline & $\begin{array}{l}\text { Mensajes } \\
\text { enviados para } \\
\text { solicitar un } \\
\text { servicio }\end{array}$ & $\begin{array}{l}\text { Mide la influencia } \\
\text { de la cantidad } \\
\text { de mensajes } \\
\text { intercambiados por } \\
\text { el agente al realizar } \\
\text { una negociación } \\
\text { cuando el agente } \\
\text { solicita un servicio } \\
\text { de otro agente }\end{array}$ \\
\hline
\end{tabular}

ejecutivas, lo que puede ser evaluado considerando la fracción de mensajes requiriendo una acción por parte del usuario o de otros agentes del sistema respecto de todos los mensajes recibidos [18].

El atributo capacidad de evolución se identifica con la capacidad del agente para adaptarse a las necesidades del entorno [24] y tomar las medidas necesarias para autoajustarse a nuevos objetivos [25]. Una buena capacidad de evolución puede ser evaluada considerando la capacidad de actualizar su estado por parte del agente, y la frecuencia de actualización de su estado [18]. La Tabla 2 presenta las seis medidas propuestas para evaluar la autonomía de un agente software.

\section{Atributos y medidas de proactividad}

La proactividad se refiere a la habilidad de los agentes en alcanzar sus metas. Para ello ha de tomar decisiones de manera dinámica (iniciativa), tener capacidad de relacionarse para recabar información que ayude a solventar los problemas (interacción) y ha de comportarse activamente ante los estímulos del entorno (reacción) [12].

El atributo iniciativa se identifica con la capacidad del agente para satisfacer los objetivos definidos en su diseño, mediante una conducta dirigida por objetivos [16], y de emprender por sí mismo una acción con la meta de alcanzar sus objetivos [2627]. Una buena capacidad de iniciativa puede ser evaluada considerando el número de roles que el agente debe desarrollar, el número de objetivos alcanzados por el agente, y la cantidad promedio de mensajes intercambiados por el agente para alcanzar sus objetivos [12].

El atributo interacción se identifica con la capacidad del agente de interactuar con otros agentes y su entorno [27]. Una buena capacidad de interacción puede ser evaluada considerando los métodos por clase definidos en el agente, y la cantidad de tipos de mensajes que pueden ser atendidos y resueltos por el agente [12].

El atributo reacción se identifica con la capacidad del agente para reaccionar a un estímulo del entorno que lo rodea de acuerdo con una conducta de estímulo/respuesta [28]. Se ha determinado que un buen autocontrol puede ser evaluado considerando la función de complejidad estructural del agente, el 
Tabla 2. Medidas características autonomía.

\begin{tabular}{|c|c|c|}
\hline \multicolumn{3}{|c|}{ Medidas de autonomía } \\
\hline \multirow{3}{*}{ Autocontrol } & $\begin{array}{l}\text { Complejidad } \\
\text { estructural }\end{array}$ & $\begin{array}{l}\text { Mide la cantidad } \\
\text { y complejidad } \\
\text { de los punteros o } \\
\text { referencias que el } \\
\text { agente usa en su } \\
\text { programación en el } \\
\text { estado interno del } \\
\text { agente }\end{array}$ \\
\hline & $\begin{array}{l}\text { Tamaño del } \\
\text { estado interno }\end{array}$ & $\begin{array}{l}\text { Mide el tamaño del } \\
\text { estado interno del } \\
\text { agente }\end{array}$ \\
\hline & $\begin{array}{l}\text { Complejidad } \\
\text { de conducta }\end{array}$ & $\begin{array}{l}\text { Mide la complejidad } \\
\text { de los servicios que } \\
\text { el agente ofrece } \\
\text { (solo aplica a } \\
\text { agentes que ofrecen } \\
\text { servicios) }\end{array}$ \\
\hline $\begin{array}{l}\text { Independencia } \\
\text { funcional }\end{array}$ & $\begin{array}{l}\text { Fracción de } \\
\text { mensajes de } \\
\text { tipo ejecutivo }\end{array}$ & $\begin{array}{l}\text { Mide la influencia } \\
\text { sobre el agente } \\
\text { de la fracción de } \\
\text { mensajes ejecutivos } \\
\text { (que solicitan una } \\
\text { acción) recibidos } \\
\text { desde el usuario, } \\
\text { al que el agente } \\
\text { representa, o de } \\
\text { otros agentes (a los } \\
\text { que está obligado a } \\
\text { responder) respecto } \\
\text { de todos los } \\
\text { mensajes recibidos } \\
\text { (considerando } \\
\text { las acciones de } \\
\text { comunicación) }\end{array}$ \\
\hline \multirow[b]{2}{*}{$\begin{array}{l}\text { Capacidad de } \\
\text { evolución }\end{array}$} & $\begin{array}{l}\text { Capacidad de } \\
\text { actualizar el } \\
\text { estado }\end{array}$ & $\begin{array}{l}\text { Mide la capacidad } \\
\text { del agente para } \\
\text { actualizar su estado }\end{array}$ \\
\hline & $\begin{array}{l}\text { Frecuencia de } \\
\text { actualización } \\
\text { del estado }\end{array}$ & $\begin{array}{l}\text { Mide el impacto } \\
\text { de la frecuencia de } \\
\text { actualización del } \\
\text { estado del agente } \\
\text { durante su ejecución }\end{array}$ \\
\hline
\end{tabular}

tamaño del estado interno del agente, y la complejidad de conducta del agente [18]. Una buena capacidad de reacción depende del tiempo de procesamiento de mensajes, y el costo del procesamiento de operaciones [12]. La Tabla 3 presenta las ocho medidas propuestas para evaluar la proactividad de un agente software y sus definiciones.

En la Figura 1 se presenta un resumen de las características, atributos y medidas propuestos para el agente software.
Tabla 3. Medidas característica proactividad.

\begin{tabular}{|c|c|c|}
\hline \multicolumn{3}{|c|}{ Medidas de autonomía } \\
\hline \multirow{3}{*}{ Autocontrol } & $\begin{array}{l}\text { Complejidad } \\
\text { estructural }\end{array}$ & $\begin{array}{l}\text { Mide la cantidad } \\
\text { y complejidad } \\
\text { de los punteros o } \\
\text { referencias que el } \\
\text { agente usa en su } \\
\text { programación en el } \\
\text { estado interno del } \\
\text { agente }\end{array}$ \\
\hline & $\begin{array}{l}\text { Tamaño del } \\
\text { estado interno }\end{array}$ & $\begin{array}{l}\text { Mide el tamaño del } \\
\text { estado interno del } \\
\text { agente }\end{array}$ \\
\hline & $\begin{array}{l}\text { Complejidad } \\
\text { de conducta }\end{array}$ & $\begin{array}{l}\text { Mide la complejidad } \\
\text { de los servicios que } \\
\text { el agente ofrece } \\
\text { (solo aplica a } \\
\text { agentes que ofrecen } \\
\text { servicios) }\end{array}$ \\
\hline $\begin{array}{l}\text { Independencia } \\
\text { funcional }\end{array}$ & $\begin{array}{l}\text { Fracción de } \\
\text { mensajes de } \\
\text { tipo ejecutivo }\end{array}$ & $\begin{array}{l}\text { Mide la influencia } \\
\text { sobre el agente } \\
\text { de la fracción de } \\
\text { mensajes ejecutivos } \\
\text { (que solicitan una } \\
\text { acción) recibidos } \\
\text { desde el usuario, } \\
\text { al que el agente } \\
\text { representa, o de } \\
\text { otros agentes (a los } \\
\text { que está obligado a } \\
\text { responder) respecto } \\
\text { de todos los } \\
\text { mensajes recibidos } \\
\text { (considerando } \\
\text { las acciones de } \\
\text { comunicación) }\end{array}$ \\
\hline \multirow[b]{2}{*}{$\begin{array}{l}\text { Capacidad de } \\
\text { evolución }\end{array}$} & $\begin{array}{l}\text { Capacidad de } \\
\text { actualizar el } \\
\text { estado }\end{array}$ & $\begin{array}{l}\text { Mide la capacidad } \\
\text { del agente para } \\
\text { actualizar su estado }\end{array}$ \\
\hline & $\begin{array}{l}\text { Frecuencia de } \\
\text { actualización } \\
\text { del estado }\end{array}$ & $\begin{array}{l}\text { Mide el impacto } \\
\text { de la frecuencia de } \\
\text { actualización del } \\
\text { estado del agente } \\
\text { durante su ejecución }\end{array}$ \\
\hline
\end{tabular}

\section{CASO DE ESTUDIO}

A continuación se presenta un caso de estudio correspondiente al proceso de gestión de agentes de un entorno bancario, que considera agentes del tipo banco y del tipo clientes, y que realiza un intercambio de conocimientos y actuaciones entre estos agentes (Figura 2). Los agentes del sistema se relacionan por medio de operaciones típicas de un banco, como son crear una cuenta, retirar dinero o pedir un crédito. El sistema consta de dos clientes y tres bancos. Los primeros iniciarán las 


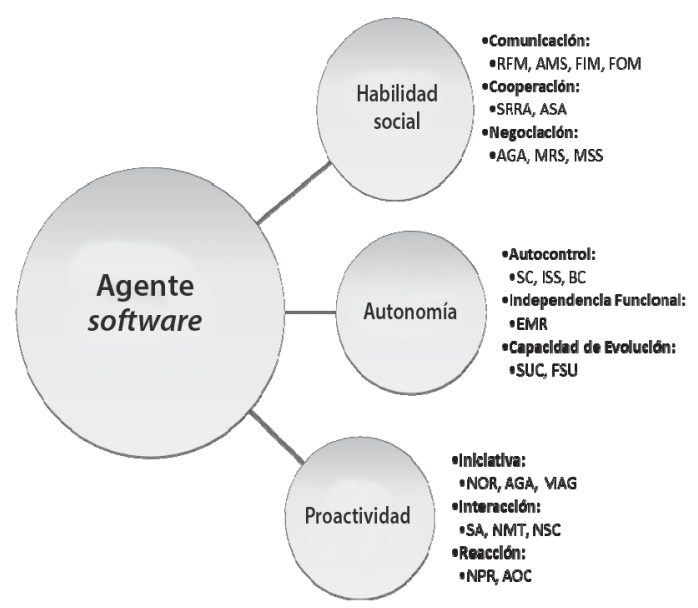

Figura 1. Características, atributos y medidas de un agente software.

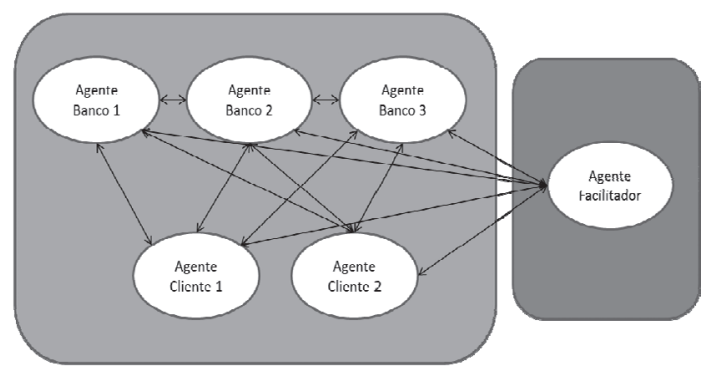

Figura 2. Agentes del sistema bancario.

conversaciones y los bancos intentarán responderlas realizando procesos internos o preguntas a otras entidades de la misma naturaleza.

Para realizar este estudio se utilizó un software que aplica en forma práctica las evaluaciones de las medidas de este trabajo [29-30]. Para diseñar el software se utilizó la plataforma JADE [31] y la codificación se realizó en el entorno de desarrollo integrado Eclipse [32]. Se realizaron tres experimentos diferentes para valorar las distintas medidas expuestas en este trabajo.

En todos los experimentos se consideró la opinión de dos expertos en el desarrollo de software orientado a agentes con una valoración entre 0 a 10 de las medidas consideradas en el sistema, y luego se aplicó el test de Kolmogorov-Smirnov² [33], en que

2 El test de Kolmogorov-Smirnov es un test no paramétrico que se utiliza para determinar la bondad de ajuste de dos distribuciones de probabilidad entre sí. El test de Kolmogorov-Smirnov se consideró la media aritmética de las opiniones de los expertos y la media aritmética de las medidas para hacerlas comparables.

En el primer experimento se aplicaron las medidas para evaluar la habilidad social de cada agente en el sistema. La Figura 3 presenta el valor de los atributos y la habilidad social, calculados considerando la media aritmética de las medidas por cada atributo. Se observa que los agentes bancarios tienen un mayor valor del atributo cooperación, mientras que los agentes clientes lo tienen en la negociación. Analizando los valores para el sistema, los valores promedios de todos los atributos son muy similares (en torno al 86\%).

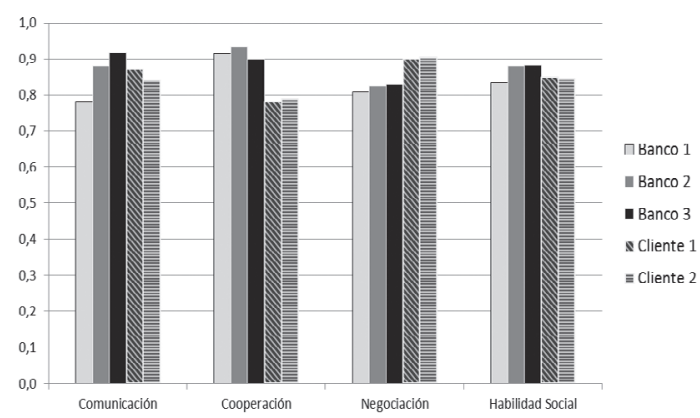

Figura 3. Promedio valores de atributos habilidad social por agente.

Finalmente, el valor de la habilidad social del sistema es $86 \%$, la que es alta para este sistema. La Figura 4 presenta una comparación de los resultados del promedio obtenido por el sistema y de los expertos, aplicando el test de Kolmogorov-Smirnov.

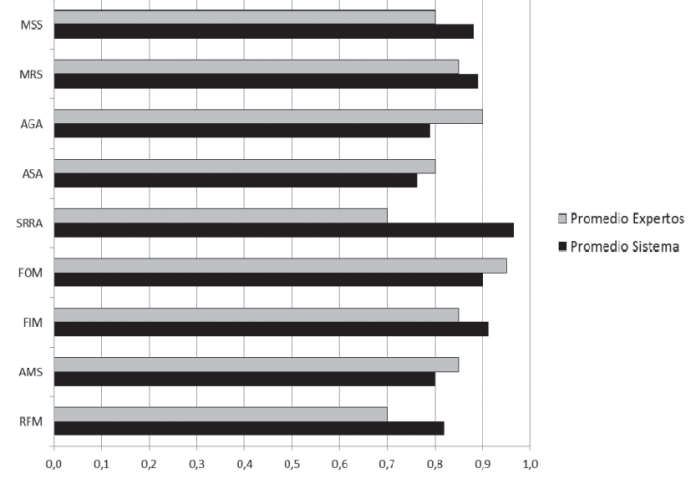

Figura 4. Valores medidas habilidad social.

considera que las muestras son similares si el valor de significancia es menor que 0,9 o 0,95 (según la exigencia del usuario). 
Respecto de la valoración de expertos sobre las medidas de habilidad social aplicando el test de Kolmogorov-Smirnov se consideró la media aritmética de las opiniones de los expertos y la media aritmética de las medidas, obteniendo un nivel de significación de 0,50 , lo que permite rechazar la hipótesis de que ambas muestras provienen de distintas distribuciones.

En el segundo experimento se aplicaron las medidas para evaluar la autonomía de cada agente en el sistema. La Figura 5 presenta el valor de los atributos y la autonomía, calculados considerando la media aritmética de las medidas por cada atributo. Los atributos influyen en el valor de autonomía de cada agente, siendo menor los de los agentes bancarios y un poco mayor el de los agentes clientes, debido a que los agentes bancarios tienen una mayor capacidad de evolución e independencia funcional. La autonomía promedio es $80 \%$.

En la Figura 6 se presenta la comparación de los resultados del promedio obtenido por el sistema y de los expertos, aplicando el test de KolmogorovSmirnov. Respecto de la valoración de expertos

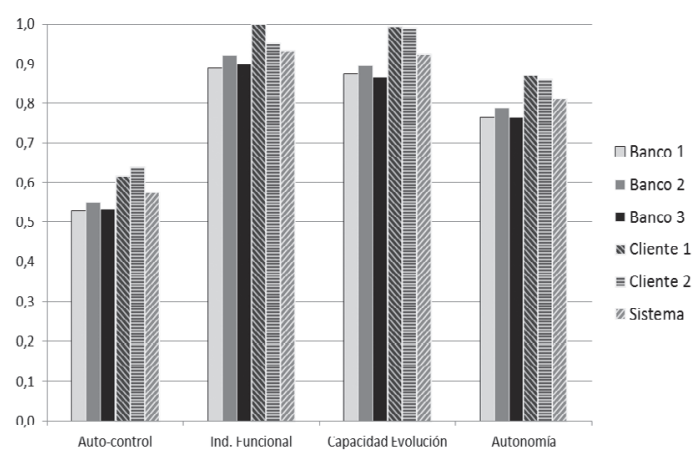

Figura 5. Promedio valores de atributos autonomía por agente.

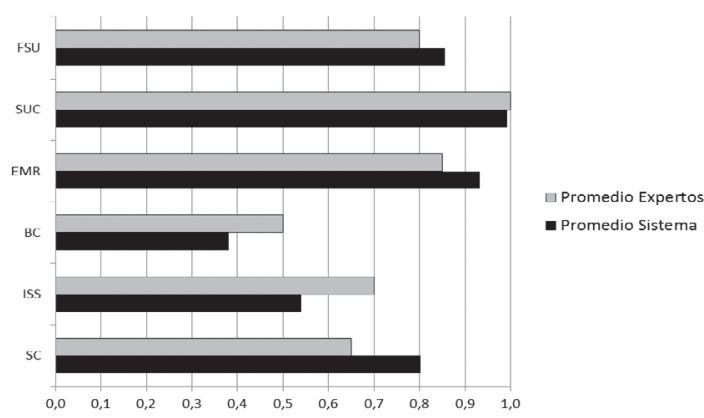

Figura 6. Valores medidas autonomía. sobre las medidas de autonomía de los agentes del sistema se consideró la media aritmética de las opiniones de los expertos y la media aritmética de las medidas, obteniendo un nivel de significación de 0,75 , lo que permite rechazar la hipótesis de que ambas muestras provienen de distintas distribuciones.

En el tercer experimento se aplicaron las medidas para evaluar la proactividad de cada agente en el sistema. En la Figura 7 se presenta el valor de los atributos y la proactividad, calculados considerando la media aritmética de las medidas por cada atributo.

Se observan altos valores de iniciativa de los agentes bancarios (en promedio es 97\%) seguido de los otros dos atributos, interacción y reacción (95\% en promedio). Finalmente, la proactividad del sistema es muy alta, con $96 \%$ en promedio, lo que se explica por la alta interacción de los agentes del sistema. En la Figura 8 se presenta la comparación de los resultados del promedio obtenido por el sistema y de los expertos, aplicando el test de KolmogorovSmirnov. Respecto de la valoración de expertos

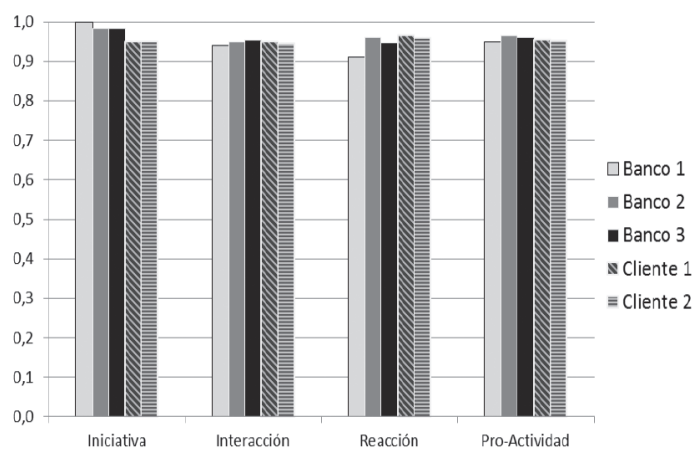

Figura 7. Promedio valores de atributos proactividad por agente.

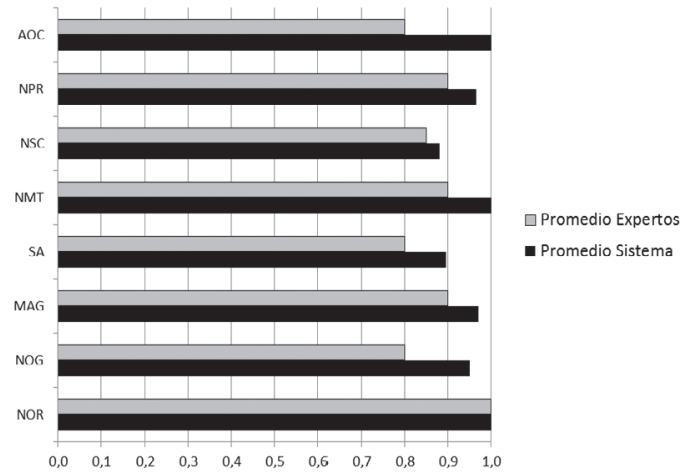

Figura 8. Valores medidas proactividad. 
sobre las medidas de proactividad de los agentes del sistema se consideró la media aritmética de las opiniones de los expertos y la media aritmética de las medidas, obteniendo un nivel de significación de 0,45 , lo que permite rechazar la hipótesis de que ambas muestras provienen de distintas distribuciones.

\section{CONCLUSIONES Y TRABAJO FUTURO}

A partir del estudio de la tecnología de agentes software se definieron distintas características que pueden estar presentes en este tipo de software. Considerando estas características se abordó el trabajo de estudiar tres de ellas: la habilidad social, la autonomía y la proactividad. Estas, a su vez, se dividieron en 9 atributos (tres por cada característica), para considerar distintos factores que representan aspectos importantes de cada característica, susceptibles de poder ser evaluados.

Para estos atributos se diseñaron 23 medidas para la evaluación de estas cualidades, y que pueden usarse en una aplicación orientada a agentes para obtener una evaluación consistente de las características estudiadas.

Se desarrolló además una aplicación [29], haciendo uso de la plataforma Eclipse [32] y de la orientación a objetos en Java, que permitió implementar una forma de evaluación automática de las medidas propuestas en este trabajo, sobre distintas aplicaciones orientadas a agentes, y que demuestra que las medidas desarrolladas son automatizables. La utilización de esta herramienta proporcionó además la posibilidad de refinar las medidas para estudiar con mayor detenimiento su comportamiento. Además, la aplicación permitía modificar los valores de los pesos asociados a las medidas para así determinar los más adecuados a cada situación en estudio.

Este trabajo constituye un avance de la investigación realizada para alcanzar un modelo de calidad en el paradigma orientado a agentes, que permita a los desarrolladores contar con una ayuda con la que evaluar satisfactoriamente su trabajo, de modo de asegurar que este sea el adecuado para las funciones para el que fue producido, considerando las características que debe cumplir cada componente de la aplicación.
Como trabajo futuro se señala determinar medidas de calidad asociadas a las restantes características del software orientado a agentes, definiendo sus atributos más relevantes, a los que asociar un conjunto de medidas que permitan evaluar dichos atributos. Además determinar medidas asociadas a la calidad del proceso de desarrollo del software orientado a agentes. Con estas medidas se podría completar un modelo de calidad que comprenda tanto las medidas de calidad del producto como las del proceso y con ello se completará un modelo de calidad que comprenda las medidas de calidad del producto y del proceso.

\section{REFERENCIAS}

[1] C. Vega, L. Rivera y A. García. "Mejores prácticas para el establecimiento y aseguramiento de la calidad de Software". Libros y Manuales, CIET, Centro de Cómputo Académico, UCC, Universidad Cristóbal Colón. 2008.

[2] J. McCall. "An Introduction to Software Quality Metrics". Software Quality Management. J.D. Cooper and M.J. Fisher (eds.). Petrocelli Books, pp. 127-142. 1979.

[3] J.L. Fuertes. "Modelo de Calidad para el Software Orientado a Objetos". Tesis para optar al grado de doctor. Facultad de Informática. Universidad Politécnica de Madrid. Madrid, España. Marzo 2003.

[4] ISO. "ISO/IEC 9126-1:2001. Software engineering- Product quality- Part 1: Quality Model". International Standard ISO/IEC 9126-1:2001. 2001.

[5] R. Dumke, R. Koeppe and C. Wille. "Software Agent Measurement and Self-Measuring Agent-Based Systems". Preprint $N^{\circ} 11$. Fakultät für Informatik. Otto-von-GuerickeUniversität. 2000.

[6] B. Far and T. Wanyama. "Metrics for AgentBased Software Development”. Proceedings of the IEEE Canadian Conference on Electrical and Computer Engineering. CCECE 2003, pp. 1297-1300. May, 2003.

[7] K. Shin. "Software Agents Metrics. A Preliminary Study and Development of a Metric Analyzer". Project Report ${ }^{\circ}$ H98010. Dept. Computer Science. School of Computing. National University of Singapore. 2003/2004 
[8] S. Zamani, R. Nassiri and S. Jabbehdari. "A New Test Process in Agent-oriented Software Engineering”. International Journal of Advancements in Computing Technology. Vol. 3, Issue 7 pp. 1-9. August, 2011.

[9] M. Wooldridge and N. Jennings. "Intelligent agents: Theory and practice". The Knowledge Engineering Review. Vol. 10, Issue 2, pp. 115152. 1995.

[10] A. Mas. "Agentes Software y Sistemas Multi-Agente: Conceptos, Arquitecturas y Aplicaciones". Pearson Educación S.A. Madrid, España. 2005.

[11] I. Duncan and T. Storer. "Agent testing in an ambient world". Proceedings of the Pervasive 2006 Workshop, pp. 757-764. Dublin, Eire. May, 2006.

[12] F. Alonso, J.L. Fuertes, L. Martínez and H. Soza. "Measuring the Pro-Activity of Software Agents". Proceedings of the Fifth International Conference on Software Engineering Advances (ICSEA 2010), pp. 319-324. Nice, France. August, 2010.

[13] H. Soza. "Medidas de Calidad para Software Orientado a Agentes". Tesis para optar al grado de doctor. Facultad de Informática. Universidad Politécnica de Madrid. Madrid, España. Febrero 2013.

[14] L. Cernuzzi and G. Rossi. "On The Evaluation Of Agent Oriented Methodologies". Proceedings of the OOPSLA 02 - Workshop on Agent-Oriented Methodologies, pp. 21-30. Seattle, USA. November, 2002.

[15] M. Huber. "Agent Autonomy: Social Integrity and Social Independence". Proceedings of the International Conference on Information Technology ITNG'07, Las Vegas, Nevada. IEEE Computer Society, pp. 282-290. Los Alamitos, CA, USA. 2007.

[16] M. Wooldridge. "An Introduction to MultiAgent Systems". John Wiley and Sons, Ltd. England. 2002.

[17] F. Alonso, J.L. Fuertes, L. Martínez and H. Soza. "Measuring the Social Ability of Software Agents". Proceedings of the 6th ACIS International Conference on Software Engineering. Research, Management \& Applications (SERA 2008), pp. 3-10. IEEE Computer Society. August, 2008.

[18] F. Alonso, J.L. Fuertes, L. Martínez and H. Soza. "Towards a set of Measures for
Evaluating Software Agent Autonomy". Proceedings of the 8th Mexican International Conference on Artificial Intelligence (MICAI 2009), pp. 73-78. Guanajuato, México. 2009.

[19] M. Bonifacio, P. Bouquet, R. Ferrario and D. Ponte. "Towards a model of goal autonomous agents". Proceedings of the Fourth International Bi-Conference Workshop on Agent-Oriented Information Systems. AOIS '02, 59, pp. 27-28. 2002.

[20] K-M. Chao, A. James and P. Norman. "A framework for Intelligent agents within effective concurrent design". Proceedings of the sixth international conference on computer supported cooperative work in design, pp. 338-341. IEEE. 2001.

[21] C. Wille, R. Dumke and S. Stojanov. "Quality Assurance in Agent-Based Systems Current State and Open Problems". Preprint $\mathrm{N}^{\circ}$ 4, Fakultät für Informatik. Universität Magdeburg. 2002.

[22] B. Far. "Software Agents: Quality, Complexity and Uncertainty Issues". Proceedings of the First IEEE International Conference on Cognitive Informatics (ICCI'02), pp. 122131. Canada, August, 2002.

[23] R. Pfeifer and C. Scheier. "Understanding Intelligence". MIT Press. September, 1999.

[24] J. Murdock. "Model-Based Reflection for Agent Evolution". Technical Report GIT-CC00-34. Doctoral Thesis. Georgia Institute of Technology. Atlanta. 2000.

[25] B. Friedman and H. Nissenbaum. "Software Agents and User Autonomy". Proceedings of the First International Conference on Autonomous Agents, pp. 466-469. 1997.

[26] D. Rousseau and B. Moulin. "Mixed initiative in interactions between software agents". Proceedings of the 1997 Spring Symposium on Computer Models for Mixed Initiative Interaction. AAAI Press, Portland, Oregon, USA. March, 1997.

[27] S. Covey. "The Seven Habits of Highly Effective People". Simon \& Schuster UK Ltd. London, 1989. 15th anniversary edition in 2004.

[28] A. Orro, M. Saba and E. Vargiu. "Using a Personalized, Adaptive and Cooperative Multi Agent System to Predict Protein Secondary Structure". Proceedings of the First International Workshop on Multi- 
Agent Systems for Medicine, Computational Biology, and Bioinformatics, BIOMED'05, Utrecht, The Netherlands, pp. 170-183. July, 2005.

[29] O. Villasante. "Evaluación de Calidad de Sistemas Multiagente: una aplicación práctica a un sistema bancario". Memoria para optar al título de Ingeniero Informático. Facultad de Informática. Universidad Politécnica de Madrid. Madrid, España. Febrero 2011.

[30] O. Villasante. "Evaluación de Calidad de Sistemas Multi-Agente para entornos JADE". Tesis para optar al grado de Master en Informática, Facultad de Informática.
Universidad Politécnica de Madrid. Madrid, España. Febrero 2011.

[31] F. Bellifemine, A. Poggi and G. Rimassa. "JADE: a FIPA2000 compliant agent development environment". Proceedings of the Fifth International Conference on Autonomous Agents, pp. 216-217. ACM. 2001.

[32] Eclipse Platform Technical Review, Object Technology International Inc., February, 2003.

[33] I.M. Chakravart, R. Laha, J. Roy: Handbook of Methods of Applied Statistics, Volume I, John Wiley. 1967. 